\title{
tamasomā jyotirgamaya: Seeking the Self Amidst Covids' Cytokine Cyclones
}

\author{
R.x. Covea and B. Mishra ${ }^{1,2,3,4,5,6^{*}}$
}

\begin{abstract}
Pondering on pandemics and the promise of purification from the plethora of problems that it has spawned, the paper builds on a game-theoretic model of host-pathogen interaction, and... moves beyond. It highlights how quickly this 'wicked' problem has led to deceptive Nash equilibria of certain information-asymmetric games as well as their sequels of more complex intertwined games at human scale but without an exit strategy in sight. In the absence of clarity (e.g., access to complete information) and yet facing a capricious and complex conspirator, we overview an exemplary solution, created by RxCovea, and examine how it might help.
\end{abstract}

\section{A Rival Viral Arrival}

All genesis stories might as well start with the picture of the three worlds that we have borrowed from Sir Roger Penrose. In our modified picture, the three worlds correspond to the physical world, symbolic world, and signaling world, assembled in an ecosystem in which one devours the next and is devoured by the one before. The picture probably had its origin in Kabbalah, as depicted by Giordano Bruno as mundus triplex in his book De Magia. Bruno's three worlds were: the archetypical world (of divine magic), the sensible world (of physical magic), and the rational world (of mathematical magic). During his lifetime, which overlapped with Galileo's, Bruno's ideas, however, remained poorly understood. In 1600 , Bruno was burned at the stake in Rome's Campo de' Fiori, after being tried for heresy by the Roman Inquisition. Like many other contemporaneous Averroistas, Bruno was seeking deeper answers: which of these three worlds represent an objective (sound and complete) truth? And how can one separate truth from falsity to find ones' true-selves-separate from non-selves? And would it converge to a stable self-hood, albeit a conventional nash equilibrium (NE)? (Fig. 1)

A minuscule subset of the visible physical world is comprised of molecules, made out of atoms, and can be modeled mathematically using tools from quantum and classical mechanics_-but not necessarily in a unified framework.
Biomolecules constitute an important subclass of molecules and are dominated by phosphorous, carbon, nitrogen, oxygen, hydrogen, etc. The biomolecules interact with each other and with water molecules (e.g., via ATP hydrolysis reaction process) It is important that the "cellularizing" ensembles of such biomolecules-very likely membrane separated from the environmentremain lively (via a sequence of endergonic and exergonic reactions) and safe (maintaining right $\mathrm{pH}$ level and avoiding $\mathrm{H}_{2} \mathrm{O}_{2}$, etc.). Because liveliness and safety conferred to the cells can be abstracted by a "utility" function, cells may be abstracted as individual "biomolecular agents" competing with each other in an evolutionary game in which the fittest survives and replicates.

These biomolecules are also able to store useful information via concatenation of ATPlike subunit monomers to form polymers (e.g., RNA, DNA, microtubules, etc.) and, thus, symbolically encode molecular patterns, each with its own utility. Such polymers form the symbolic world, in which they duplicate (or allow transcription and translation into other polymers mapping homomorphic patterns) and propagate the information stored in molecular patterns. If the duplication process permits variations and a utility-dependent replicator dynamics and pattern-specific information propagation is unidirectional, it sets the stage up for a Darwinian evolution and amplifies diversity, giving rise to
Mathematics and CS Courant Institute of Mathematical Sciences, NYU, 251 Mercer Street New York, NY 10012, USA

${ }^{2}$ CSE, Tandon School of Engineering. New York USA.

${ }^{3}$ Cell Biology, NYU

School of Medicine, New York, USA.

${ }^{4} \mathrm{QB}$, Cold Spring Harbor Lab, New York, USA.

${ }^{5}$ Oncology, New York Genome Center, New York, USA.

${ }^{6}$ Human Genetics, Mt. Sinai School of Medicine, New York USA

*mishra@nyu.edu 
Cytokine: A signaling molecule, acting as a chemical messenger released by white blood cells, including macrophages, monocytes, or lymphocytes. The cytokines include the interferons, the interleukins, tumor necrosis factor, etc.

HLA: The human leukocyte antigen (HLA) complex is a group of related proteins, encoded by the major histocompatibility complex (MHC) in humans. HLA genes are highly polymorphic which means that they have many different alleles, allowing them to fine-tune the adaptive immune system—differently for different individuals. HLAs corresponding to MHC class I (A, B, and C) present peptides from inside the cell. Foreign antigens presented by MHC class I attract $\mathrm{T}$ lymphocytes called killer $\mathrm{T}$ cells.

Memory T cell: Persistent T cells that bear a receptor for a specific antigen that was previously encountered in the course of illness or vaccination. It plays a role of a verifier to ensure safe execution of the signaling game.

Interferons: A family of proteins (lymphokines), secreted by infected host cells to protect uninfected cells from viral infections.

Antibody: An important part of the adaptive immune system. It is a protein usually in the blood and destroys or neutralizes bacteria, viruses, or other harmful toxins. Antibody production takes place in response to antigen. They are members of a class of proteins known as immunoglobulins, produced by B cells in response to stimulation by an antigen.

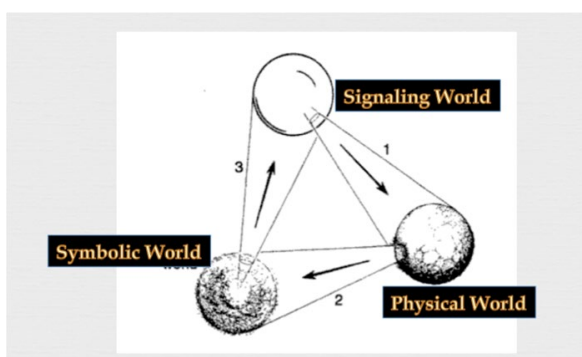

Figure 1: Molecules, molecular patterns, and (pathogen/damage) associated molecular patterns: who signals who? We depict the three worlds, important to defining a host's self and protected by a complex immune system, but amenable to invasion by a simple deceptive virus which, in turn, can be deceived by a vaccine We develop a signaling game framework to study virology, and immunology-while we hope to extend the framework to asymptomatic patients, with misaligned utilities, and impaired by moral hazards in economy. We suggest that they all conspire to make the Covid problem a "wicked" (difficult or impossible to solve) problem. Courtesy R.x. Covea et al.

inter-agent information asymmetry. Thus, not all possible molecular patterns would be uniformly (or information-symmetrically) represented in the symbolic world; only an infinitesimally small subset of all possible patterns would encode viable genomes (DNA), transcriptomes (RNA), or translatomes/proteomes (proteins).

Interactions among biological macro-molecules may be described using a sender-receiver signaling structure, where an expressed macromolecule, such as a protein or RNA, constitutes a signal sent on behalf of a sender agent (e.g., cell). The signal may be modeled as the threedimensional conformation and physicochemical properties of the macro-molecule. A receiver agent (e.g., another macro-molecule) may then bind to the signal macro-molecule, which produces an action (such as an enzymatic reaction). The action produces utility for the participating agents, sender and receiver, and thereby-albeit indirectly-a change in overall fitness of the organism, and hence its genome (in evolutionary game theory, utility and fitness are treated as analogous). When there is common interest, the utility is expected to benefit both sender and receiver and their selectivity, thus driving Darwinian co-evolution. If there is neither common identity (i.e., they are "strangers") nor common interest (i.e., they pose "dangers"), the sender and receiver may avoid each other in order not to undermine their common fitness. Thus, evolution of processes encoding, recognizing, and acting against PAMP (Pathogen Associated Molecular Patterns) and DAMP (Damage-Associated Molecular Patterns) plays an important role in the evolution of the multicellular immune systems. Note that not all strangers are dangerous (e.g., symbionts such as corona virus in bats) and that some non-strangers could be dangerous (e.g., cytokine storms inducing activated white blood cells).

However, since the immune systems also play an important role in defining many more higher order evolutionary processes: e.g., HLA diversity, mate-selection, cognitive processes, culture, etc., our very human intellectual and cultural journey has also shaped our secular scientific understanding of the physical universe, albeit, it still remains inconsistent and lacks unification (e.g., quantum and classical mechanics). The resulting body of scientific literature, however, forms only a small part of our body of human knowledge that relates us to a tentative, though conventional, picture of a 'natural world,' containing both symbolic and signaling systems.

As humans struggle with their understanding of the genesis and mitigation of a surging pandemic, initiated by a single zoonosis event in a remote Asian town, it is worthwhile to meditate on these connections among the three worlds-mundus triplex, further subdivided and re-connected by such subdisciplines as virology, immunology, epidemiology, logical/mathematical inference, and economics-going from nanoscale viruses to global-scale human societies.

Or, "must we wring the neck of a certain system in order to stuff them into [] pigeon holes for the satisfaction of the analogy mongers:" Do the worlds divide neatly into Bruno's three magics—sciences into three disciplines: physics, mathematics, and divine? Or does it just model a biological Gödelian self-referential self-seeking, selfishly greedy computation, hopelessly aspiring to halt with stable (sound and complete) Turing decidability notion(s) of self(-ves)? Can it reach an NE (Nash equilibria), in which self separates from non-self, decoy epitopes from neutralizing, susceptible from infected, symptomatic from asymptomatic, essential economic activities from dispensable?

\section{Evolutionary Signaling Game}

Replicator dynamics allow the signaling game to be couched in evolutionary terms ${ }^{6}$. Replicator dynamics arise from the increased replication of players with higher utility (fitness). Thus, if a 


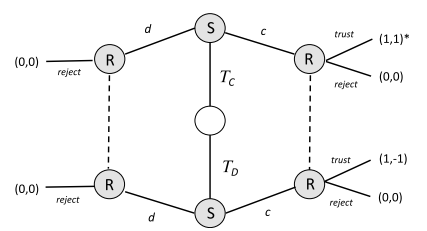

Figure 2: Extensive Form Signaling Game describing biomimicry by a virus to fool the immune system: cell can be of two types $T_{C}$ (self) or $T_{D}$ (non-self viral): they can send $c$ signals honestly or by mimicry, respectively, and may be trusted or rejected by the immune system; similarly, they can send $d$ signals erroneously or honestly, respectively, and may be rejected by the immune system. Courtesy S. Massey et al.

cell has a strategy that results in increased utility, then it will increase in frequency in a population. For a sender cell, this would entail sending a signal that may result in an increased utility, while, for a receiver cell, this would entail undertaking an action that likewise may result in an improved utility (e.g., growth rate) (Fig. 2).

As already suggested, these dynamics represent a process analogous to Darwinian (adaptive) evolution or positive selection. In other words, as part of the innate immune system, sender agent, a dendritic cell (DC), in a multicellular organism that recognizes an appropriate DAMP/PAMP combination could signal receiver agent, a macrophage $(M \Phi)$, to engulf and digest foreign substances (associated with the molecular patterns, AMP) in an action process called phagocytosis. The innate system is said to be engaged in a signaling game, in which DC is the sender; $M \Phi$, the receiver; they signal using cytokines and phagocytosis which is the action taken by the receiver upon receiving the signal. Their utility is determined by protection which they confer against non-self (mostly danger, potentially posed by strangers) and tolerance for self. Since, many pathogens (e.g., SARSCoV-2) can engage in deception (e.g., entering a host cell by targeting host ACE2 receptorby binding to a site somewhat distant from the ACE2 canonical binding site), innate immune system may need other auxiliary adaptive agents to be "educated" ex tempore, replicate dynamically and retain memory. These cells belonging to the adaptive immune system are not strategic (e.g., do not confer fitness along the germ line), but play an important role as recommenders ( $B$ cells)and verifiers ( $T$ cells) in taming the deceptive pathogens that can grow and mutate rapidly to frustrate the innate immune system.
However, in an adversarial chase, pathogens can evolve strategies to frustrate the education of $\mathrm{T}$ cells (e.g., antigen presentation), but can be remedied by costly signaling via DC's maturation and chemo-taxis to germinal centers. It is no wonder, most immunology jokes end with the punchline ${ }^{7}$, "The immunologist says, 'The thing is, the immune system is very complicated...' And the cardiologist says, 'Just shoot me now."

We hope to simplify the model by formulating the innate immune system as a signaling game ${ }^{4,}$, which, though prone to deception, can be critically tamed by the education, surveillance, and memory acquired by the adaptive immune system; the education of the adaptive immune system, though highly costly, can also be hijacked by a deceptively simple virus and points to a need for a better understanding of how the system behaves among various hosts (bats and humans with diverse HLA types).

Signaling games Signaling games are multiplayer (usually, two players) games with incomplete information: specifically, one player is informed and the other player is not. The informed player's strategy set consists of signals contingent on information; uninformed player's strategy set consists of actions contingent on signals.

Game is played as follows

1. Player $S$ is assigned a type $t \in T$

2. $S$ send to player $R$ a signal $s \in M$.

3. $R$ takes an action $a \in A$.

Payoff function The utility functions for sender and receiver are dependent on the type of the sender, signal sent and action carried out by the receiver:

$$
u^{i \in\{S, R\}}: T \times M \times A \rightarrow \mathbb{R} .
$$

Equilibrium concept

Behavior strategies for senders and receivers:

1. For $S$, a function $\mu: T \times M \rightarrow[0,1]$, such that:

$$
\sum_{s \in M} \mu(t, s)=1, \text { for all } t .
$$

$\mu(t, s)=$ Probability that $S$ with type $t$ sends signal $s$.

2. For $R$, a function $\alpha: M \times A \rightarrow[0,1]$, such that:
Antigen: A substance antagonizing the immune system to produce antibodies.

Antigen presenting cell (APC): A white blood cell that engulfs foreign bodies, breaks them down, and carries characteristic antigen peptides to its surface. The foreign antigen, complexed with MHC I or II (specific to host's HLA type), is presented to CD4 or CD8 to initiate an immune response specific to that peptide.

Dendritic cell: An antigenpresenting immune cell. They have elongated, tentacle like branches to trap foreign objects.

Macrophage: A large scavenger cell that ingests degenerated cells and secretes messenger proteins (monokines) involved in inflammatory reactions, lymphocyte activation, and acute systemic immune responses.

Phagocytosis: The consumption and destruction of foreign materials by white blood cells like macrophages.

CD-4 cell: "Helper" T-cell, coordinates much of the adaptive immune response.

B-lymphocytes (B cells): B-lymphocytes are blood cells derived from the bone marrow and spleen involved in the production of antibodies. $B$ cells produce antibodies, when primed by $\mathrm{T}$ cells. B-cell lymphocytes can later differentiate into plasma and memory cells. In our signaling game, they are modeled as recommenders and accelerate convergence to a separating NE based on the past memory of encounters.

T cells (T lymphocytes): A thymus derived white blood cell that precipitates a variety of cell-mediated immune reactions. Three fundamentally different types of $\mathrm{T}$ cells are recognized: helper, killer, and suppresser (each has many subdivisions). They can be modeled as verifiers in the signaling game. 


$$
\sum_{a \in A} \alpha(s, a)=1, \text { for all } s .
$$

$\alpha(s, a)=$ Probability that $R$ takes action $a$ following signal $s$.

We may then say that the receiver may acquire a subjective probability distribution over $T$ :

$$
\beta(t, s)=\frac{\mu(t, s) \pi(t)}{\sum_{t^{\prime} \in T} \mu\left(t^{\prime}, s\right) \pi\left(t^{\prime}\right)} .
$$

\subsection{Nash Equilibria}

Proposition Behavior strategies $(\alpha *, \mu *)$ form a Nash equilibrium iff for all $t \in T$ :

$\mu(t, s)>0$ implies

$$
\begin{aligned}
& \sum_{a \in A} U^{S}(t, s, a) \alpha(s, a) \\
= & \max _{s^{\prime} \in S} \sum_{a \in A} U^{S}\left(t, s^{\prime}, a\right) \alpha\left(s^{\prime}, a\right) ;
\end{aligned}
$$

\& for all $s \in S$ (s.t. $\left.\sum_{t \in T} \mu(t, s) \pi(t)>0\right)$

$$
\begin{aligned}
\alpha(s, a)>0 \text { implies } & \\
& \sum_{t \in T} U^{R}(t, s, a) \beta(t, a) \\
= & \max _{a^{\prime} \in A} \sum_{t \in T} U^{R}\left(t, s, a^{\prime}\right) \beta\left(t, a^{\prime}\right) .
\end{aligned}
$$

Signaling games in nature To assume reasonable utility functions, we use two natural maps that relate the sender's private information to receiver's action that would be desirable via Rate Distortion functions combining $I(\cdot, \cdot)$ (mutual information or rate) with a distortion $d^{\cdot}(\cdot, \cdot)$ combined using suitable Lagrange parameters $\lambda$.

- Mapping Types and Actions into Signals:

- $f^{S}: T \rightarrow A ; \quad f^{R}: A \rightarrow T$.

- Sender:

- $\quad U^{S}=I(T, M)+\lambda_{S} d^{S}\left(f^{S}(t), a\right)$.

- Receiver:

- $\quad U^{R}=I(A, M)+\lambda_{R} d^{R}\left(t, f^{R}(a)\right)$.

\subsection{Deception}

The model of signaling games The game thus involves two players, namely:

$$
\begin{aligned}
& S-\text { Sender (Informed) } \\
& R-\text { Receiver (Uninformed) . }
\end{aligned}
$$

- Their roles can be shared; $S$ may only have partial information; and $R$ may be allowed to perform distributed actions.

- A notion of TYPE, $T$ private to $S$, is captured by a random variable $t$ whose support is given by $T$ (known to sender $S$ ). $\pi(\cdot)=$ probability distribution over $T$ is a prior belief of $R$ that the sender's type is $t$.

\section{Subjective reality}

- Note that the distribution of signals received by $R$ is given by the probability distribution $\pi_{M}$, where:

- $\pi_{M}(s)=\sum_{t \in T} \pi_{T}(t) \mu(s \mid t)$,

- And the distribution of actions produced by $R$ is given by the probability distribution $\pi_{A}$, where:

- $\quad \pi_{A}(a)=\sum_{s \in M} \pi_{M}(s) \alpha(a \mid s)$.

- Clearly, $\pi_{T}$ and $\pi_{A}$ are probability distributions on $T$ and $A$, respectively. If $\hat{\pi}_{T}$ is the probability distribution on $T$ induced by $\pi_{A}$ under the function $f_{R}$, then:

$$
\hat{\pi}_{T}(\cdot):=\pi_{A}\left(f_{R}^{-1}(\cdot)\right) .
$$

A natural choice of measure of deception may be given by the relative entropy between the probability distributions $\pi_{T}$ and $\hat{\pi}_{T}$ :

Deception : $=$ Rel. Entropy $\left(\hat{\pi}_{T} \mid \pi_{T}\right)$

$$
=\sum_{t \in T} \hat{\pi}_{T}(t) \log _{2} \frac{\hat{\pi}_{T}(t)}{\pi_{T}(t)} .
$$

This definition describes deception from the point of view of the receiver. To get the notion of deception from the point of view of the sender, one needs to play the game several rounds.

Thus, Signaling Games may reach a Nash equilibrium that is stable but not deception-free (e.g., immune system may reach a homeostasis that may not be ideal in separating self from non-self).

- If the game reaches a Separating Equilibrium then: Each type $t$ sends a different signal $M_{t}$. $f^{S}: t \mapsto a\left[M_{t}\right] \ldots$ 


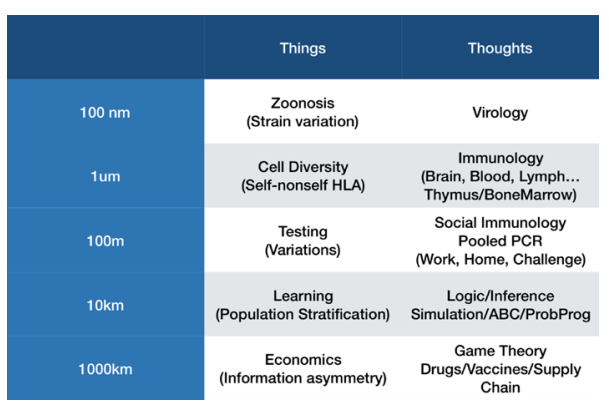

Figure 3: Covid poses a wicked problem affecting different human organ, physical, and socia systems at different spatio-temporal scales. Note: Wiki defines "a wicked problem as a problem that is difficult or impossible to solve because of incomplete, contradictory, and changing requirements that are often difficult to recognize. Another definition is 'a problem whose social complexity means that it has no determinable stopping point.' Moreover, because of complex inter-dependencies, the effort to solve one aspect of a wicked problem may reveal or create other problems.' Covid problem started as a healthcare problem, but quickly evolved into an economic problem, triggering various issues related to humans' fundamental rights. Courtesy: R.x. Covea et al.

- If, on the other hand, the game reaches a Pooling Equilibrium: all types $t$ send a single signal $s *$ with probability 1 .

- Convention and deception The divergence between the objective probabilities and the subjective probabilities induced by conventional equilibria.

- Solution Costly signaling; credible and noncredible threat; aligned utilities; augmenting with additional non-strategic playersdenoted as: $m$ Recommenders $+n$ Verifiers

The possibility of deception (e.g., biomimicry by SARS-CoV2) in the Nash equilibrium (NE) of the signaling game may be the reason why the Innate immune system (with PAMP and DAMP) is not the ultimate solution; the NE must be stable, but also keep up with rapidly evolving pathogens. It leads to a need for an adaptive immune system, training of $\mathrm{T}$ cell by $\mathrm{DC}$, chemo-taxis, and maturation processes that DC must go through, cytokine storms, etc. Therefore, "it is very complicated.." and more so if we need to create a vaccine that must deceptively mimic the virus, which may have been already deceptively mimicking the host system, and the vaccine must be carefully analyzed and regulated so as not to catalyze autoimmune disorders or antigen-dependent enhancement ADE.

\section{Quo Vadis: Covid}

Covid-19 poses a wicked problem. "Why does the repeated game it plays-Covid game-have long and branching chains, intertwined hierarchies, plenty of room for deception, and explosive growth rates? Why not use similar features, perhaps, needed in the counter measures to tame it ${ }^{1}$ ?-" A proportionally rapid and rigorous analysis of the game as well as synthesis of hyper-local policies (e.g., strategies) addressing molecular, individual, and community-wide actions. For argument's sake, assuming that it only manifests as a socio-economic question, it remains unfathomable what economic utilities might be ethically and equitably traded off for loss and depreciation of human capital (Fig. 3).

Many of the unknown unknowns are exacerbated by the impacts of unquantified-or unquantifiable-(1) proportion of asymptomatic patients, (2) comorbidities and vulnerability of various subpopulations, (3) co-evolution of the disease in the presence of others (flu or multiple strains-with unknown cross immunity), (4) number and combinatorial structure of the network of super-spreaders, (5) individual boundedly rational compliance, (6) spatio-temporal reaction-diffusion nature of the disease (e.g., Turing Labyrinth for herd immunity), (7) accuracy, frequency, and scaling of biomedical tests (separating asymptomatic from symptomatic, susceptible from infected and infected from immune, etc.), (8) unpredictability of treatment and preventive measures such as socialdistancing, contact-tracing, repeated lock-downs, and quarantines, (9) allocation of resources for patients across vulnerabilities, demographics, genders and races, etc. RxCovea has sought to develop and openly disseminate mathematical and computational tools and theories to address these problems with the help of modelers (SDEs, graph-based, hybrid, cellular automata), game theorists (signaling games, minority games), logicians (formal methods, model checkers, belief revision), algorithmicists (multiobjective decision and optimization problems), statisticians (bandit problems, reinforcement learning), and computational systems biologists (in silico vaccine

\footnotetext{
${ }^{1}$ L. Rudolph: personal comm. 2020
} 
and drug discovery). Detailed Game-Theoretic analyses in different contexts, namely, germline, somatic, or population evolution, may be found elsewhere $2,3,5$.

(1) $100 \mathrm{~nm}$ scale: Virology-A task group ("la famiglia"), consisting of Salvatore Alaimo, Eva Bischof, Jantine Broek, Ashley Duits, Alfredo Ferro, Naomi Maria, Alfredo Pulvienti, and- Valentina Rapicavoli, has been studying the systems biology of the virus and host-pathogen interactions. The group has been able to replicate in silico-safely, scalably and inexpensively-a large class of in vivo and in vitro experiments to test a substantial number of hypotheses regarding host-pathogen-drug interactions. Rigorous statistical analysis, to correct for multiple hypotheses testing, then points to the strategies (possibly involving deceptive biomimicry) that the virus may evolve and how drugs may be re-purposed to enable the host organs, systems, and pathways to counter safely. The group also aims to investigate how SARS-CoV2 virus achieves a symbiotic existence with other reservoir hosts (e.g., bats and pangolines) and how its genome mutates in order that hosts' immune system may accommodate such a ("pooling")

\footnotetext{
${ }^{2}$ In YACHT's simplest incarnation:

A user is recommended a pool in which their saliva can be tested within a time interval:

If the user selects to be pool-tested and the pool-test result is negative, the user is given a badge (non-counterfeitable plastic wrist tag, encrypted electronic badge or a crypto-coin tag) that lasts a pre-determined period. During that period with the safe badge, the user may be allowed to enter (resp. exit) certain safe (resp. unsafe) locations.

If, on the other hand, the user selects to be pool-tested and the pool-test result for the user is positive, then the user is given an unsafe badge - and recommended a uniformly randomly selected pool (Hot-Potato protocol) and/or exponentially randomly selected time interval (Exponential-Back-off protocol) for being retested. The user without a safe badge is virtually quarantined from entering a safe region (or leaving unsafe region-which must have sheltering-in-place or hospitals).

Also, since the subsequent choices of the pools are (by recommendation) independent, if the user is truly negative, they will be determined so (almost surely) no later than a fixed run-length of "(apparent) false-positive" results. If a user fails to be negative after fixed run-length of "(apparent)-true-positive" results, the user must be quarantined.
}

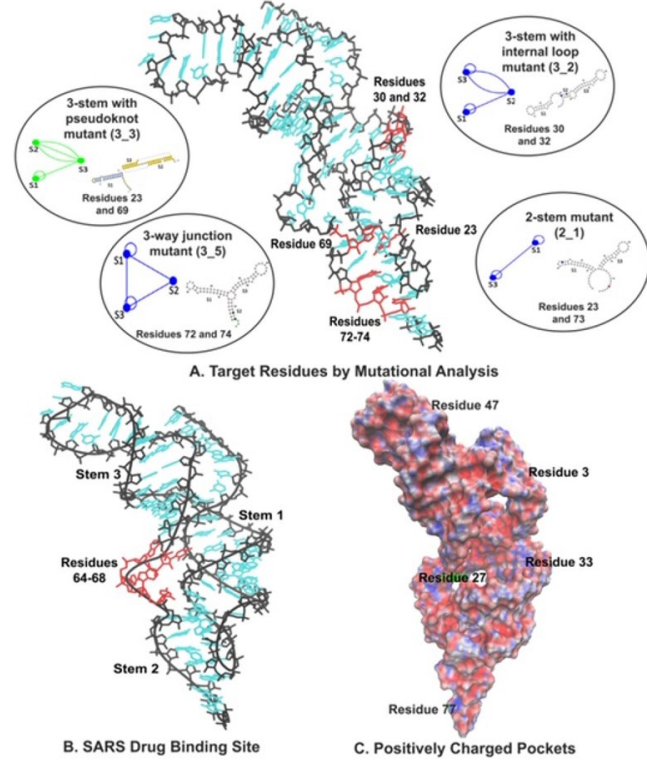

Figure 4: Target RNA residues for anti-viral therapy in the frame-shifting element of SARS-CoV-2 determined by molecular modeling. a Shows the $3 \mathrm{D}$ structure of the frame-shifting element of SARS-CoV-2 as modeled by MD (molecular dynamics) with the key residues which are identified by destroying the pseudoknot and/or a stem using graph theory and genetic algorithms. The identified residues are then used in the drug screening algorithm. Other identified residues are shown in $\mathbf{b}$ and $\mathbf{c}$. Courtesy: T. Schlick et al.

Nash equilibria (perhaps, with constrained antigenic drift). Another task group ("the gamers") consisting of William Casey and Steven Massey has developed models of biomimicry (especially the ones achieved by SARS-CoV-2, mimicking Polybasic Cleavage Site, PCS mutations to interact with host furins) that speaks to intelligent and safe vaccine and drug design. Charles Cantor studies sophisticated multicolor PCR testing methods, tracking the viral genomic mutations. Marco Antoniotti studies the phylogeny of the genomic strain mutations. Tamar Schlick with her molecular modeling group studies the structure and dynamics of the FSE (frame-shifting element) of the viral RNA genome and its screening in silico to discover novel anti-viral therapy to inhibit protein synthesis (Fig. 4).

(2) $1 \mu \mathrm{m}$ scale: Immunology—another task group ("the adaptors") has been studying the host immune system's response to viral 
infection: the group consists of James Bannon, Charles Cantor, Ashley Duits, John Conolloy, Kelly Ganjei, Jia Han, and Mike Lotze, and it analyzes longitudinal adaptomic data from patients, using temporal network analysis using advanced combinatorics and formal methods. It is desired, with the availability of adequate resources, to expand the team to study the genomic markers stratifying the probands, who exhibit a wide range of symptoms-asymptomatic vs symptomatic or super-spreaders or Kawasaki/MISC disease phenotypes. It is conjectured that the immunogenic biomarkers can be characterized by a GWAS (GenomeWide Association Study), if certain genomic regions (e.g., $p$-arm of $\mathrm{Chr} 6$ ) are mapped haplotypically with long-range single-molecule technology such as nanomapping. Possible biomarkers clustering around HLA, KIR, and TLR regions are being studied by a group consisting of Marcin Imilienski, Sanjan Kumar, Jason Reed, and Amir Toor. Vijay Chandru and Ramesh Hariharan are aiming to initiate a similar project in India, which enjoys a more interesting admixture population, driven by endogamy ("the grandest genetic experiment ever performed on man," according to Theodosius Dobzhansky).

(3) $100 \mathrm{~m}$ scale: Epidemiology/Social Immunology-RxCovea's third task group ("the labyrinthers") has focused on various epidemiological models and their parametric control using clever (pool- and individual-) testing methods with low latency: the group comprises Ved Basu, Jantine Broek, Shireshendu Chatterjee, Inavamsi Enaganti, Hilary Gao, Eva Guo, Mathew Jacobs, and Kim Mishra (for modeling); Charles Cantor, Vijay Chandru, Manoj Gopalakrishnan, Inavamsi Enaganti, Satyajit Mahrana, and Rohit Nandwani (for testing); the two subgroups cross-pollinate intimately. The subgroups started with standard Cellular Automata (CA) agent-based model for incorporating geographical distributions of infection and easily captures the notion of discrete pairwise interactions, since the new state of a Cellular Automaton (CA) is based on its current state and the states of its surrounding CAs as well as some common evolving rules. The infection spreads from an infected CA to adjacent neighboring CAs, while infected patients also recover and become immune to the reinfection. We have assumed the following states for the population Exposed, Symptomatic, Asymptomatic, Susceptible, Recovered/Immunefurther augmented with such other states as: unknown, tested, vaccinated, quarantined, retested, recurrent, etc., with respect to other SARS-CoVs and flu. RxCovea has also implemented CTMC (continuous time Markov chain) and realistic SDE (stochastic differential equation) models - the later model obtained using Turing's reaction-diffusion equation framework and the analysis of its solutions-e.g., Turing labyrinths. We conjecture that the formation of Turing Labyrinths (possibly further stratified by HLAs and Cov2-strains) will play a role in the formation of herd immunity, vaccination, and social-distancing policies. These pattern formations can be further moulded by RNA, antigen, and immunoglobulin tests (YACHT, Yet Another Covid-Health Testing) ${ }^{2}$, as well as contact tracing (with 1 or 2 degrees of separation) both operated at a large scale with a selected periodicity. The original YACHT design motivated by the following game-theoretic model: (i) Repeated Game: Repeated saliva pool testing (involving hundreds of individuals in a pool) using rapid and accurate (duplicated) PCR test is feasible with negligible false negatives and manageably low false positive. (ii) Private Game/ No Intermediary: Sample collection must remain safe, unsophisticated, ubiquitous, and unbiased (from self-selection) and, thus, may not involve robotics and technicians. (iii)Decentrality: Data collection and storage must be reliable, private, and yet not centralized. (iv) Factual and Counter-factual: The analysis is flexible, hypotheses-testable, easyto-communicate, and adaptive in controlling compliance, prevalence, immunization, and resource usage. YACHT's resemblance to Ethernet's Aloha protocol and PageRank's random surfer approach, especially with small/moderate prevalence, adds to our faith in its robustness. We have simulated a model, with promising results, that partition the population into two regions ("home" encountering both safe and unsafe household members and "work" encountering safe non-household members) and assumes that the household statistics differ from country to country (India, Afghanistan, USA, and The Netherlands). In the near future, we wish to resimulate the system with one additional region: "challenge region," which may be factual or counter factual (e.g., with vir-
Immunoglobulin: A protein that acts as an antibody to fight off pathogens. There are five classes: $\operatorname{IgG}, \operatorname{IgA}, \operatorname{IgD}$, IgM, and IgE. Recombinant and pooled immunoglobulins from blood donations are being used to fight off SARSCoV-2 infection.

Neutralizing antibody: An antibody that neutralizes (renders harmless) the infectivity of microorganisms, particularly viruses. 
T helper cells: Lymphocytes responsible for assisting other white blood cells in responding to infection, processing antigen, and triggering antibody production (also known as T4 cells and CD4 cells).
T killer cells: A major component of cytotoxic lymphocyte response (CTL), responsible for lysing infected or cancerous cells, and $\mathrm{T}$ killer cells (not to be confused with natural killer cells) are a subset of CD8+ lymphocytes.
T suppressor cells: T lymphocytes responsible for turning the immune response off after infection is cleared, a subset of CD8+ lymphocytes. tual viral strains and spreading and testing mechanisms) and allow collection of statistical data for hypotheses testing (Fig. 5).

(4) $100 \mathrm{~km}$ scale: Logic, Inference, and Learning-The fourth task group ("the model checkers") is a data-science group to delineate the causal structures among various pandemic-related events; the group includes: Marco Antoniotti, Jantine Broek, Daniel Cage, Will Casey, Shirshendu Chatterjee, Vijay Chandru, James Edmondson, Yaron Gvili, Stella Luna, Ramon Luna, and Larry Rudolph. The group is preparing for a near future when massive amount of meta-data, (e.g., involving pools, mobility, and geographic occupancy datafrom FourSquare) can be combined with Machine Learning tools augmented with Formal Methods to hypothesize and test causal relationship and intervention strategies. Since our games include complex subgames involving credible and non-credible threats, monitoring human behavior-without assuming perfect rationality-is likely to play a critical role. We plan to use Probprog tools (Julia/Turing ${ }^{3}$ ) to understand the disease etiology, and individuals' strategic choices tempered by the knowledge of causality, time, logic and games, privacy, reputation, trust, deception, and cellularization (Fig. 6).

5) $1000 \mathrm{~km}$ Scale: Economics and Normative Equilibria-The "Open Sim," group (including Ved Basu, Jantine Broek, Will Casey, Shirshendu Chatterjee, and Yaron Gvili) is studying how to connect components of global data to infer effect of stratification of populations based on demographics, age, gender, genomics (e.g., HLA), (co-)morbidity, etc. to understand genuine causal structures only using natural experimental data and without getting bogged down by the bias due to multiple hypotheses testing and Simpson's effect. The economic roles played by the disrupted interrelationships among different subgroups (and their possible simulation by digital means, when individuals need to be socially distanced) have a strong impact on the $\mathrm{V}$ or $\mathrm{W}$ shaped economic recovery. The "Burpa group" (comprising Kose John, Samir Saadi, and Cheryl Qi) has designed a novel market microstructure to enable health

\footnotetext{
${ }^{3}$ A probabilistic programming software package, named after the computer scientist and logician Alan Turing.
}
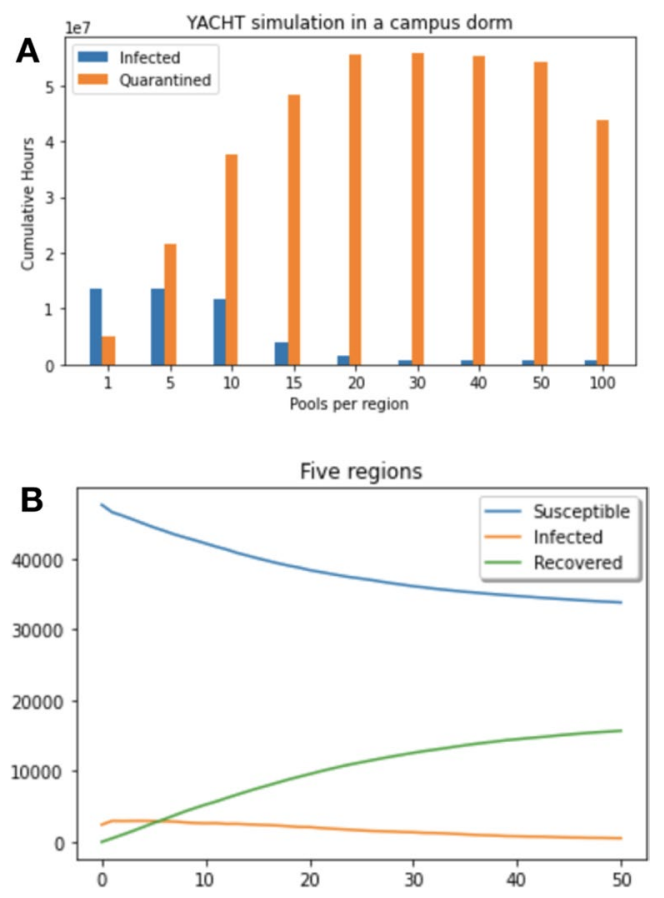

Figure 5: YACHT's performance on a university campus with five dorms. The student population is simplified to be heterogeneous with two assumed immunogenic biomarkers: e.g., HLA types HLA' and HLA", where agents of type HLA' are less infectious than HLA" agents. Simulation assumes that there is only one viral strain. a Observe that increasing pool sizes decreases the total number of infections while increasing quarantining. After a delay, the quarantined time also reduces. b Shows the SIR plot of the same simulated system with exactly 15 pools per dorm. Observe that the prevalence is easily controlled to below 5\%. Courtesy I. Enaganti et al.

insurance for a patient population (social network) interacting with scientists and clinicians (expert network) to create digital healthcare markets that tame its risks by combining multiple SPV's (Special Purpose Vehicles) and securitization (Fig. 7).

\section{Pavamana (Purification)}

In a more optimistic future, to which we hope to return soon, RxCovea will focus on better algorithms for randomly surfing explainable AIs to create a world-wide adaptive immune system to protect humans and things physiologically, physically, and digitally. The adaptive learning recommenders and verifiers will be able to anticipate and create mitigating plans for future zoonotic events. For now, our collective afforts aim to 


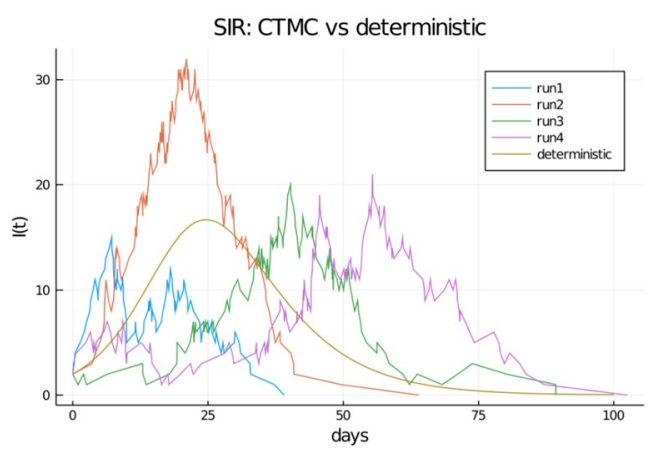

Figure 6: Epidemiological Model-e.g., compartmental models such as SIR with compartments: S (susceptible), I (infected), and R (removed). The diagram shows why the uncertainties in parameters may make the models unpredictable-especially, when population is of finite size. Still, the models can be used fruitfully to stress test various plausible policies and affect boundedly rationa agent behaviors. Courtesy: J. Broek et al.

From Babbling lead me to Separating equilibria!

\section{असतो मा सद्वमय ।}

तमसो मा ज्योतिर्गमय ।

मृत्योर् मामृतं गमय ।

From falsehood lead me to truth, From darkness lead me to the light, From death lead me to immortality.

Figure 7: pavamāna abhyāroha: purification prayers; The mantra was originally meant to be recited during the introductory praise of the Soma sacrifice by the patron.

provide computational models of host-pathogen interactions that will help to define parameters critical to healthcare policies and methods to control the viral spread- "the hammer and the dance." We hope that optimistic future will also include a successful completion of Human Haplotypic HLA Genome project, making it unfashionable among immunologists to say, "The thing is, the immune system is very complicated... !".

\section{Publisher's Note}

Springer Nature remains neutral with regard to jurisdictional claims in published maps and institutional affiliations.

\section{Acknowledgements}

In mid-March of 2020, spurred by a hypothesis centered around HLA-hetero-zygotic advantages of asymptomatic Covid patients, we organically converged to form the "cure COVid-19 for Ever and For All"(RxCovea) Group, a rigorous community of scientists, clinicians, AI specialists, mathematical and computational/data modelists, pharmaceutical, public and digital health intelligence representatives from multiple institutions, countries, and scientific training. Within the network, self-organized task forces continue to conduct core projects, structured around hypotheses, and minimal viable products (MVP's), mentored, and examined by experienced senior members.

Special "thank you"s to Ashley, Charles, Larry, Lex, and Vijay-privately "typed" as friends, mentors, and mentees-who meticulously avoid social-distancing in all the three worlds: The paper has benefited a lot from their signals and actions!

The contributions by BM were supported by NSF Grants CCF-0836649 and CCF-0926166, NCI Physical Sciences-Oncology Center Grant U54 CA193313-01, and a US Army grant W911NF1810427. The contributions by $\mathrm{RxC}$ have been voluntary and unsupported. The work of TS (T. Schlick) was supported by NSF RAPID Award 2030377 and NIH Award R35GM122562 (NIGMS).

Received: 22 August 2020 Accepted: 3 September 2020 Published online: 22 October 2020

\section{References}

1. Beckett S (1929) 'Dante... Bruno. Vico.. Joyce', in Finnegans Wake: A Symposium - Exagmination Round His Incamination of Work in Progress [... \&c.]. Shakespeare \& Co., Paris 1929; facs. rep. edn. New Directions, NY 1972, pp 1-22

2. Casey W, Kellner A, Memarmoshrefi P, Andre Morales J, Mishra B (2019) Deception, identity, and security: the game theory of sybil attacks. Commun ACM 62(1):85-93

3. Casey W, Morales JA, Wright E, Zhu Q, Mishra B (2016) Compliance signaling games: toward modeling the deterrence of insider threats. Comput Math Organ Theory 22(3):318-349

4. Huttegger SM, Skyrms B, Smead R, Zollman KJS (2010) Evolutionary dynamics of lewis signaling games: signaling systems vs. partial pooling. Synthesis 172(1):177-191

5. Loohuis LO, Witzel A, Mishra B (2014) Cancer hybrid automata: model, beliefs and therapy. Inf Comput 236:68-86

6. Skyrms B (2010) Signals. Oxford University Press, Oxford

7. Yong E (2020) Immunology is where intuition goes to die. The Atlantic 


\section{Appendix: Immune System Glossary}

The immune system is a host defense system comprising many biomolecular structures and signaling processes within an organism that protects against pathogens, dangerous (and possibly, stranger) to the organism-from viruses to parasitic worms, and distinguish them from the organism's own healthy tissue. It is possible to study the immune systems as a signaling game whose Nash equilibria separate two types: self and non-self. The immune system consists of an innate system coupled with an adaptive system: both following replicator dynamics, but innate system selecting over organisms' germ line and adaptive system adapting over organisms' somatic immunological memory. [Adapted from https:// morgridge.org/outreach/teaching-resources/virol ogy-immunology/immune-system-glossary/].

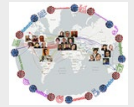

R.x. Covea The group (RxCovea) benefits from the experience of Gideon Berger (Sr. MD, Black Stone), Daniel Cage (CEO, SpeedChain), Charles Cantor (Founder of the Human Genome Project, ex-CTO Sequenom), Vijay Chandru (Co-Founder, Strand), Ashley Duits (Red Cross Blood Bank Foundation Curaçao, Willemstad, Curaçao and Curaçao Biomedical \& Health Research Institute, Willemstad, Curaçao), Inavamsi Enaganti (Department of Computer Science, Courant Institute, NYU), J. Kelly Ganjei (Cognate BioServices Inc), Jian Han (Founder iReportoire), Ramesh Hariharan (CEO, Strand Life Sciences), Zurit Levin (CEO, Compugen) Mike Lotze (Asst. Vice Chancellor, Prof. Immunology, UPMC), Sultan Meghji (CEO, Neocova), Thomson Nguyen (Entrepreneur-in-Residence, KleinerPerkins), Stelios Papadoupolous (Chairman Biogen), Larry Rudolph (VP, Two Sigma), Tariq Samad (Senior Fellow, Technology Leader Institute, Ex-Honeywell), Tamar Schlick (Prof., NYU), Lex van der Ploeg (CEO, Yao - the Bard, LLC), Chris Wiggins (Prof, Columbia, CDO, NYT), and Alex Zhavoronkov (Founder/CEO of InSilico), among 150 others, give or take a few.

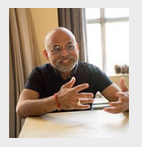

B. Mishra An educator, an inventor, as well as a mentor to technologists, entrepreneurs, and scientists. Prof. Mishra founded the NYU/Courant Bioinformatics Group, a multidisciplinary group working on research at the interface of computer science, applied mathematics, biology, biomedicine, and bio/ nano-technologies. He is the author of a textbook on algorithmic algebra and more than 200 archived publications. He has advised and mentored more than 35 graduate students and post-docs in the areas of computer science, robotics and control engineering, applied mathematics, finance, biology, and medicine. He holds 21 issued and 23 pending patents in areas ranging over robotics, model checking, intrusion detection, cyber security, emergency response, disaster management, data analysis, biotechnology, nanotechnology, genome mapping and sequencing, mutation calling, cancer biology, fintech, adtech, Internet architecture, and linguistics. Prof. Mishra's pioneering work includes: first application of model checking to hardware verification; first robotics technologies for grasping, reactive grippers, and work holding; first single molecule genotype/haplotype mapping technology (Optical Mapping); first analysis of copy number variants with a segmentation algorithm, first whole-genome haplotype assembly technology (SUTTA), first clinical-genomic variant/base calling technology (TotalRecaller), first single-molecule single-cell nanomapping technology, etc. Prof. Mishra has a degree in science from Utkal University, in Electronics and Communication Engineering from IIT, Kharagpur, and MS and $\mathrm{PhD}$ degrees in computer science from Carnegie-Mellon University. He is a fellow of IEEE, ACM, and AAAS, EAI, a fellow of National Academy of Inventors (NAI), a Distinguished Alumnus of IIT (Kharagpur), and an NYSTAR Distinguished Professor. 